\title{
Transthoracic Ultrasound for the Categorization of Pleural Effusions as Malignant: An Adjunct, but Not the Answer?
}

\author{
Coenraad F.N. Koegelenberg ${ }^{a} \quad$ Gregory Calligaro $^{b}$ \\ a Division of Pulmonology, Department of Medicine, Stellenbosch University and Tygerberg Academic Hospital, \\ and ${ }^{b}$ Division of Pulmonology, Department of Medicine, University of Cape Town and Groote Schuur Hospital, \\ Cape Town, South Africa
}

The detection, quantification and assessment of the nature of pleural effusions and the evaluation of associated pleural thickening and tumors remain the main indications for the use of transthoracic ultrasonography by respiratory physicians $[1,2]$. The echogenicity of malignant pleural effusions is known to vary and is therefore considered nonspecific, but the ultrasonographic appearance of the pleura itself may often suggest a neoplastic etiology [2].

Several morphological criteria, based on those previously validated for contrast-enhanced computed tomography (CECT) [3], were evaluated in a landmark study by Qureshi et al. [4]. The authors found that a radiologist, blinded to clinical and other radiological investigations, was able to correctively categorize 26 of 33 effusions as malignant and 19 of 19 as benign in patients referred under suspicion of malignancy. Ultrasound had an overall sensitivity of $79 \%$, specificity of $100 \%$, positive predictive value (PPV) of $100 \%$ and negative predictive value (NPV) of $73 \%$ [4]. The presence of parietal pleural thickening $>10 \mathrm{~mm}$, diaphragmatic nodularity or thickening $>7 \mathrm{~mm}$, visceral pleural thickening and pleural nodularity/irregularity were associated with malignancy. It was, however, suggested that these results may not be generalizable, given the high level of expertise at the author's tertiary referral center and the low percentage of patients with benign disease [5].

\section{KARGER}

E-Mail karger@karger.com

www.karger.com/res
In this issue of Respiration, Bugalho et al. [6] report the findings of a prospective, observational study examining the diagnostic value of transthoracic ultrasonography for predicting malignancy in undiagnosed pleural effusions in a relatively large, unselected study population. Patients were scanned by chest physicians with expertise in thoracic ultrasound who were blinded to clinical and radiological details. Static images and video clips were reviewed by at least three chest physicians with similar expertise in order to generate a consensus opinion. A total of $133 \mathrm{pa}-$ tients with pleural effusion of unknown etiology were included; 66 were eventually diagnosed with malignant effusion and 67 had benign disease.

Transthoracic ultrasound had an overall sensitivity of $80.3 \%$, specificity of $83.6 \%$, PPV of $82.8 \%$ and NPV of $81.2 \%$ for pleural malignancy.

The study confirmed the strong association between malignant effusions and the presence of pleural and diaphragmatic nodularity (OR 29.02 and 95\% CI 7.65110.01). However, unlike the report by Qureshi et al. [4] (where the pretest probability of malignancy was higher), these features were not $100 \%$ specific for malignant disease and were also found in $9 \%$ of benign effusions due to tuberculosis or pneumonia. The presence of chest wall invasion, a peripheral lung lesion associated with the effusion or hepatic metastasis were pathognomonic for malignancy, while air bronchograms and a septated pattern
(C) 2014 S. Karger AG Basel

0025-7931/14/0874-0265\$39.50/0
Coenraad F.N. Koegelenberg

Division of Pulmonology, Department of Medicine Stellenbosch University and Tygerberg Academic Hospital PO Box 19063, Tygerberg, 7505, Cape Town (South Africa) E-Mail coeniefn@sun.ac.za 
to the pleural fluid were more common in nonmalignant effusions.

Even when the ultrasonographic features of malignancy were considered in combination, thoracic ultrasound had a less than optimal diagnostic accuracy for malignant pleural effusion. One in five malignant pleural effusions were missed by thoracic ultrasound, and there was also no ultrasonographic sign with a sufficiently high NPV to obviate the need for further investigations in benign effusions. Despite its simplicity, convenience and safety, thoracic ultrasound did not have an entirely satisfactory rulein or rule-out value for the diagnosis of malignant pleural effusions. As correctly stated by the authors, the relevance of these findings for the diagnostic algorithm of unselected patients with undiagnosed pleural effusion is uncertain. A more pragmatic approach may have been to evaluate the incremental value of transthoracic ultrasound in patients once the analysis of the pleural fluid analysis had been completed. In our opinion, it remains a valuable adjunct that may direct the nature of subsequent investigations, but it does not obviate the need for additional imaging or the pursuit of a histopathological diagnosis in effusions where the pleural fluid cytology is negative and where obvious evidence of metastatic or extrapleural spread is lacking.

It should also be emphasized that CECT has superior specificity for pleural malignancy, provides additional information about the lung parenchyma, mediastinum and distant sites, and remains the preferred imaging modality for the pleura in general [5]. Correlations between CECT and thoracic ultrasonographic findings were not examined in this study. Current guidelines for the investigation of unilateral effusions generally limit the use of transthoracic ultrasound for guiding thoracocentesis and other investigations [7]. As an imaging modality, its appeal lies in the fact that it allows for the acquisition of additional, potentially useful information during routine scanning of the chest prior to invasive procedures $[1,2]$ without exposing the patient or operator to radiation. It provides this at very little extra cost (both in terms of actual healthcare costs and time), but, based on current evidence, should not be viewed as a 'stand-alone' imaging modality [4-6]. Furthermore, the question of whether or not transthoracic ultrasonography would perform equally well in 'real life' - and specifically in the hands of clinicians with less experience - remains unanswered, as most institutions will not have access to a panel of chest physicians with the level of competency of the operators in this study (at least 5 years' experience and an average of 450 exams per year).

Despite the limitations, the findings of Bugalho et al. [6] should provide further encouragement for chest physicians to achieve level 2 competency in transthoracic ultrasonography (as defined by the Royal College of Radiologists) and to use this modality in everyday practice [8]. Apart from providing an obvious real-time guide to pleural procedures, it can provide ancillary information that may direct the need for and mode of pleural tissue sampling, particularly if ultrasound findings suggest pleural malignancy [9]. The current study should also encourage units with sub-level 3 competency to report findings. Only multicenter studies could confirm the 'real' diagnostic accuracy of pleural ultrasound for malignancy.

\section{References}

1 Volpicelli G, Elbarbary M, Blaivas M, Lichtenstein DA, Mathis G, Kirkpatrick AW, Melniker L, Gargani L, Noble VE, Via G, Dean A, Tsung JW, Soldati G, Copetti R, Bouhemad B, Reissig A, Agricola E, Rouby JJ, Arbelot C, Liteplo A, Sargsyan A, Silva F, Hoppmann R, Breitkreutz R, Seibel A, Neri L, Storti E, Petrovic $\mathrm{T}$; International Liaison Committee on Lung Ultrasound (ILC-LUS) for International Consensus Conference on Lung Ultrasound (ICC-LUS): International evidencebased recommendations for point-of-care lung ultrasound. Intensive Care Med 2012;38: 577-591.

-2 Koegelenberg CF, von Groote-Bidlingmaier F, Bolliger CT: Transthoracic ultrasonogra- phy for the respiratory physician. Respiration 2012;84:337-350.

-3 Leung AN, Muller NL, Miller RR: CT in differential diagnosis of diffuse pleural disease. Am J Roentgenol 1990;154:487-492.

4 Qureshi NR, Rahman NM, Gleeson FV: Thoracic ultrasound in the diagnosis of malignant pleural effusion. Thorax 2009;64:139143.

5 Medford AR, Entwisle JJ: Thoracic ultrasound in malignant pleural effusion: a real world perspective. Thorax 2009;64:1005.

-6 Bugalho A, Ferreira D, Dias SS, Schuhmann M, Branco JC, Marques Gomes MJ, Eberhardt $\mathrm{R}$ : The diagnostic value of transthoracic ultrasonographic features in predicting malignan- cy in undiagnosed pleural effusions: a prospective observational study. Respiration 2014;87:270-278.

7 Hooper C, Lee YC, Maskell N: Investigation of a unilateral pleural effusion in adults: British Thoracic Society Pleural Disease Guideline 2010. Thorax 37 2010;65(suppl 2):ii4ii17.

8 The Royal College of Radiologists: Ultrasound Training Recommendations for Medical and Surgical Specialties, ed 2. London, The Royal College of Radiologists, 2012.

$\checkmark 9$ Koegelenberg CF, Diacon AH: Pleural controversy: closed needle pleural biopsy or thoracoscopy - which first? Respirology 2011;16: 738-746. 